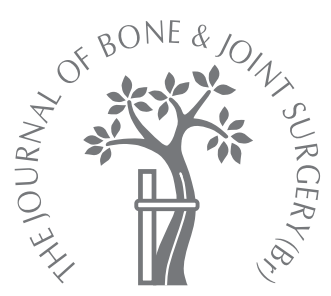

O. Levy, J. Relwani, T. Zaman, T. Even, B. Venkateswaran, S. Copeland

From Reading Shoulder Unit, Reading, England

= O. Levy, MD, MCh(Orth), Professor, Orthopaedic

Surgeon

- J. Relwani, FRCS (Tr \& Orth),

Shoulder Fellow

I. Taman, FRCS (Tr \& Orth)

Shoulder Fellow

- T. Even, MD, MSC (Stats),

Medical Statistician

B. Venkateswaran, FRCS (Tr

\& Orth), Shoulder Fellow

- S. Copeland, FRCS,

Consultant Orthopaedic

Surgeon

Reading Shoulder Unit, Royal Berkshire Hospital, Reading

RG1 5AN, UK.

Correspondence should be sent to Professor O. Levy; e-mail: oferlevy@readingshoulderunit. com

(C)2008 British Editorial Society of Bone and Joint Surgery doi:10.1302/0301-620X.90B7. $19918 \$ 2.00$

$J$ Bone Joint Surg [Br] 2008;90-B:893-8.

Received 6 July 2007;

Accepted after revision 26

February 2008

\title{
Measurement of blood flow in the rotator cuff using laser Doppler flowmetry
}

The aim of this study was to define the microcirculation of the normal rotator cuff during arthroscopic surgery and investigate whether it is altered in diseased cuff tissue.

Blood flow was measured intra-operatively by laser Doppler flowmetry. We investigated six different zones of each rotator cuff during the arthroscopic examination of 56 consecutive patients undergoing investigation for impingement, cuff tears or instability; there were $\mathbf{3 3 6}$ measurements overall.

The mean laser Doppler flowmetry flux was significantly higher at the edges of the tear in torn cuffs (43.1, 95\% confidence interval (CI) 37.8 to 48.4 ) compared with normal cuffs (32.8, $95 \% \mathrm{Cl} 27.4$ to $38.1 ; p=0.0089$ ). It was significantly lower across all anatomical locations in cuffs with impingement $(25.4,95 \% \mathrm{Cl} 22.4$ to 28.5$)$ compared with normal cuffs $(p=0.0196)$, and significantly lower in cuffs with impingement compared with torn cuffs $(p<0.0001)$.

Laser Doppler flowmetry analysis of the rotator cuff blood supply indicated a significant difference between the vascularity of the normal and the pathological rotator cuff. We were unable to demonstrate a functional hypoperfusion area or so-called 'critical zone' in the normal cuff. The measured flux decreases with advancing impingement, but there is a substantial increase at the edges of rotator cuff tears. This might reflect an attempt at repair.

Degenerative lesions of the rotator cuff are among the most common causes of pain and reduced shoulder function. ${ }^{1-5}$ Vascular impairment of the supraspinatus tendon leading to microcirculatory disturbances may predispose to such lesions. ${ }^{5}$ Post-mortem examination by Determe et $\mathrm{al}^{6}$ demonstrated relative hypovascularity near the critical site of degenerative lesions and subsequent ruptures. The microvascular pattern of the diseased cuff was studied in vivo by Biberthaler et $\mathrm{al}^{5}$ using orthogonal polarisation spectral imaging to determine quantitative measurements of the functional capillary density and diameters. However, there are no studies looking at the vascularity of the normal cuff in vivo.

The laser Doppler flowmetry technique measures blood flow in very small blood vessels, such as the low-speed flow in capillaries close to the skin surface and underlying arterioles and venules involved in the regulation of skin temperature. ${ }^{2-6}$ The thickness of the tissue sampled is typically $1 \mathrm{~mm}$, the capillary diameter $10 \mu \mathrm{m}$, and the velocity spectrum $0.01 \mathrm{~mm} / \mathrm{s}$ to $10 \mathrm{~mm} / \mathrm{s}$. This technique is physiological, permits continuous measurement, and is minimally invasive. It adds approximately four minutes to operations involving the rotator cuff.

The technique depends on the Doppler principle, whereby low-power light from a monochromatic stable laser, for example a HeliumNeon gas laser or a single-mode laser diode, is scattered by moving red blood cells, and as a consequence, is frequently broadened. This light, together with laser light scattered from static tissue, is photodetected and the resulting photocurrent processed to provide a measurement of blood flow. Where laser light is scattered in tissue with a low concentration of red blood cells, the average Doppler frequency shift is proportional to the average speed of red blood cells.

The Moor Instruments moorLAB laser Doppler monitor (Moor Instruments Ltd., Millway, Devon) uses laser radiation generated by a semiconductor laser diode operating at a wavelength of $780 \pm 10 \mathrm{~nm}$ and a maximum accessible power of $1.6 \mathrm{~mW}$. The laser light is coupled into an optical fibre within which it is transmitted to the skin or other tissue. Light is emitted as a diverging beam from the optical probe. The power output from this laser aperture is typi- 
Table I. Demographic characteristics of the study population

\begin{tabular}{llll}
\hline Group & Number of cases (\%) & Male:Female ratio & Mean age in yrs (range) \\
\cline { 3 - 3 } Impingement & $32(57.1)$ & $19: 13$ & $49.1(20$ to 75$)$ \\
Tear & $14(25.0)$ & $7: 7$ & $64.2(43$ to 75$)$ \\
Normal & $10(17.9)$ & $9: 1$ & $30.4(20$ to 48$)$ \\
All cases & $56(100)$ & $35: 21$ & $49.6(20$ to 75$)$ \\
\hline
\end{tabular}

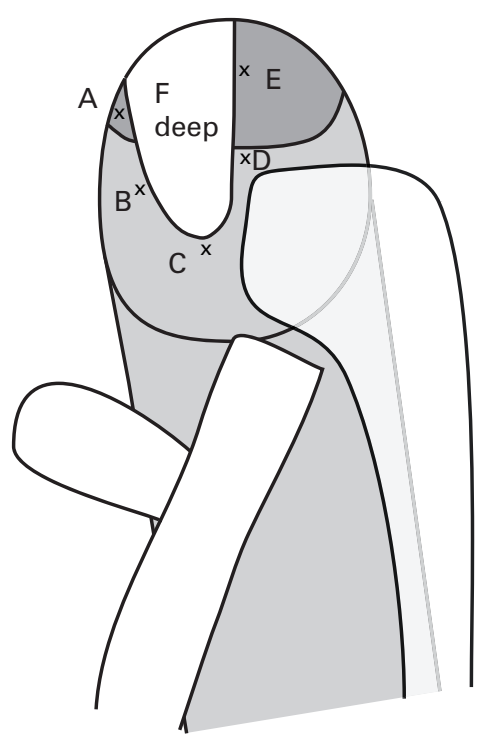

Fig. 1

Diagram showing the six points where flux measurements were made.

cally $0.5 \mathrm{~mW}$ to $1.5 \mathrm{~mW}$, with an angular spread of approximately $26^{\circ}$. The flux, an arbitrary unit of measurement of perfusion, is related to the product of the average speed and concentration of moving red blood cells in the tissue sample volume. It is proportional to the blood flow, ${ }^{7,8}$ and is the parameter most widely reported in laser Doppler publications.

The aims of our study were to clarify the value of this instrument for quantitative analysis of the microcirculation of patients during arthroscopic procedures, to determine the pattern of flux in the normal rotator cuffs and to investigate alterations in the microcirculation adjacent to degenerative lesions compared with that of an unaffected control group.

\section{Patients and Methods}

This prospective study was performed after approval by the local ethics committee, and written informed consent was obtained from all participants. A total of 56 patients (35 men and 21 women) undergoing arthroscopy for impingement (32), cuff tear (diseased cuff, 14) or shoulder instability (normal cuff, 10) were enrolled (Table I). The latter ten patients represented a control group The mean age was
Table II. Anatomical points where blood flow was measured using the laser Doppler flowmetry probe

\begin{tabular}{ll}
\hline Point of measurement & Location \\
\hline A & Anterolateral cuff, at greater tuberosity level \\
B & Anterior midpoint \\
C & Musculotendinous junction \\
D & Posterior midpoint \\
E & Posterolateral cuff, at greater tuberosity level \\
F & Deep (joint side) cuff layers \\
\hline
\end{tabular}

49.6 years (20 to 75 ). Traumatic and large/massive tears of the cuff were excluded. This study was carried out in accordance with the World Medical Association Declaration of Helsinki, last amended by the World Medical Association in Edinburgh in 2000.9

The patients were anaesthetised using a laryngeal mask and general anaesthesia. No local or regional blocks were used. The patients lay in the lateral position with the arm abducted to $30^{\circ}$. Traction of $6 \mathrm{lb}$ was applied only after measurements were taken. Arthroscopy was undertaken through a standard posterior portal. A pressure- and flowcontrolled pump (FMS Duo, Nice, France) allowed saline irrigation without any vasoactive drugs. An additional portal was placed approximately $3 \mathrm{~cm}$ lateral to the anterior edge of the acromion in line with the anterior border of the clavicle.

Following diagnostic bursoscopy, the diseased cuffs were subgrouped into mild (B1), moderate (B2), and severe (B3 cuff tear) grades of impingement according to the Copeland-Levy classification. ${ }^{10}$ The arthroscopy, grading and placement of the probe were undertaken by one of the two senior authors (SC, OL). The flux was recorded over 30 seconds at each of six points (Fig. 1 and Table II). Five of these were in the cuff over an area of $4 \mathrm{~cm}^{2}$ from the insertion at the greater tuberosity.

A MP needle probe (Moor Instruments Ltd, Millway, United Kingdom) (Fig. 2) was used for the measurements. This comprises a hypodermic stainless steel tube of external diameter $0.8 \mathrm{~mm}$ and length $80 \mathrm{~mm}$. Separation of the fibre centres (the surface area of light collection by the needle) is $0.25 \mathrm{~mm}$. The probe was inserted using an 18-gauge needle as a conduit through the skin and soft tissues.

The moorLAB laser Doppler monitor (Moor Instruments Ltd) was connected to a personal computer through a USB 


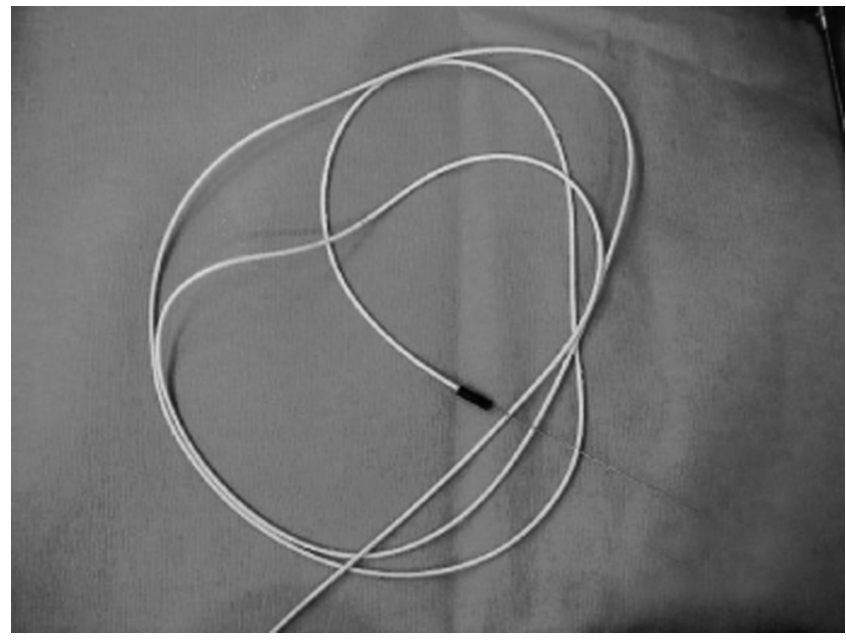

Fig. 2

Photograph of the MP4 needle probe.

port for data collection and analysed using a dedicated Window-based package, moorSOFT (Moor Instruments Ltd) for Windows/moorLAB.

During the measurements, the arthroscopic pump was arrested and the valve of the arthroscope opened to ambient air pressure to ensure conditions of physiological pressure within the subacromial space. In order to maintain stable macro-haemodynamic parameters, the mean arterial pressure was controlled by a standard non-invasive pressure device and maintained at $70 \mathrm{mmHg}$ by the anaesthetist throughout the measurement. The $\mathrm{CO}_{2}$ levels were kept below $5.0 \mathrm{kPa}$. The ambient light effect was eliminated by turning the arthroscopy illumination to a minimum during the readings and confirming that it did not interfere with any measurement. As movement between the tip of the probe and the tissue being measured causes a Doppler shift, thereby producing blood flux artefacts, the surface of the probe was carefully fixed and held to that of the cuff, with the lead secured to prevent unwanted artefact noise. Before each reading, it was confirmed that there was no abnormal blood pooling within the area being measured, as this would have affected measurements of flux.

The values obtained from the cuff disease group were compared with those of the patients with shoulder instability and normal cuffs who also underwent arthroscopic measurements (control group). The outcome variable was the mean flux over a period of 30 seconds. Predictor variables included the patients' age, gender, the location of measurement (A, B, C, D, E or F, Fig. 1 and Table II) and the study group (impingement, cuff tear, or normal). Multiple linear regression analysis was carried out to assess how each of these variables might affect the outcome of laser Doppler flowmetry flux, having accounted for all other predictors. The mean measurements of flux in the groups were compared and tested using the $t$-test and analysis of variance
(ANOVA). Statistical analysis was carried out using SAS release 8.2 (SAS Institute Inc., Cary, North Carolina). A value of $\mathrm{p}<0.05$ was considered significant.

\section{Results}

In total, 336 measurements were recorded in six anatomical locations in each shoulder. Using multiple linear regression analysis across all 336, with the outcome variable of the flux, and the predictors being age, gender, location and the study group, it was observed that only the study group was a significant predictor of the outcome (F-test, $\mathrm{p}<0.0001)$. Age, gender and anatomical location were not significant predictors of flux (F-test, $\mathrm{p}=0.1606, \mathrm{p}=0.2116, \mathrm{p}=3520$, respectively).

A highly significant difference in blood flow was observed among the study groups with the mean and $95 \%$ confidence interval (CI) for the laser Doppler flowmetry flux as 25.4 (95\% CI 22.4 to 28.5 ) in impingement, 43.1 (95\% CI 37.8 to 48.4$)$ with a cuff tear and $32.8(95 \%$ CI 27.4 to 38.1 ) in the normal cuff ( $\mathrm{p}<0.0001$, one way ANOVA; Table III, Fig. 3).

The normal rotator cuff did not show a clear 'critical' zone of hypoperfusion in the lateral (insertional) part of the rotator cuff in this study. In considering 60 readings from the ten control cuffs, there was no statistically significant variation in flux within the six areas measured ( $t$-test, $p=0.2370)$, and therefore the so-called critical zone of hypoperfusion in the rotator cuff was not demonstrated.

When the samples from the six anatomical locations in the cuff were pooled for analysis, the blood flow was lower in impingement than in normal cuffs $(t$-test, $\mathrm{p}=0.0196)$. It was higher at the edges of the tear in torn than in normal cuffs ( $t$-test, $\mathrm{p}=0.0089)$, and significantly lower in those with impingement than in those with a tear ( $t$-test, $\mathrm{p}<0.0001)$.

Analysis of the mean laser Doppler flowmetry flux and 95\% CI in the normal, impingement and tear groups by anatomical location showed the same trend of reduced blood flow in impingement compared with normal, and an increase in flow at the edges of the tear in torn cuffs compared with the normal cuffs. Statistical significance was achieved only for zones $\mathrm{A}$ and $\mathrm{E}$ (the anterolateral and posterolateral positions in the cuff at the level of the greater tuberosity level; Fig. 4).

\section{Discussion}

Laser Doppler flowmetry of the microcirculation has been used in clinical medicine to evaluate and monitor microcirculation in various tissues, including bone. ${ }^{8,11-15}$ In the upper limb, it has been used to assess autonomic dysfunction in frozen shoulders. ${ }^{16}$ It has also been used to measure blood flow in the supraspinatus muscle during isometric contraction, ${ }^{17}$ and for continuous percutaneous measurement of the microcirculation of skeletal muscle at varying levels of force of contraction as determined electromyographically. ${ }^{18}$ However, to our knowledge this is the first study using laser 
Table III. Blood flow (mean laser Doppler flowmetry flux) across all six anatomical locations by cuff disease $(p<0.0001$, one-way ANOVA)

\begin{tabular}{lllcc}
\hline \multirow{2}{*}{ Group } & Number of measurements & Blood flow & \\
\cline { 3 - 5 } & & Mean (95\% confidence interval) & Minimum & Maximum \\
\hline Impingement & 192 & $25.4(22.4$ to 28.5$)$ & 2.2 & 123.0 \\
Tear & 84 & $43.1(37.8$ to 48.4$)$ & 10.0 & 114.0 \\
Normal & 60 & $32.8(27.4$ to 38.1$)$ & 7.0 & 89.1 \\
Overall & 336 & $31.2(28.7$ to 33.7$)$ & 2.2 & 123.0 \\
\hline
\end{tabular}

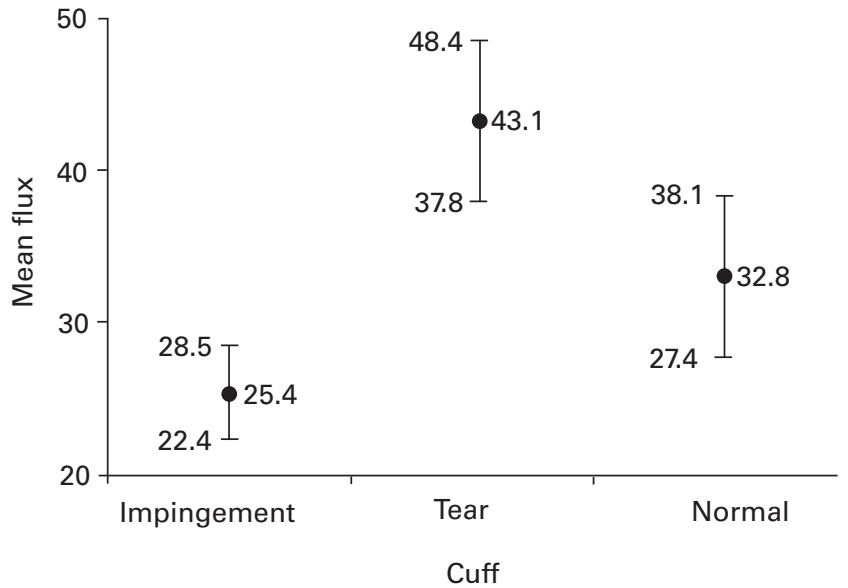

Fig. 3

Graph showing the mean and 95\% confidence interval for laser Doppler flowmetry flux by cuff disease.

Doppler flowmetry to investigate the normal and pathological tendons of the rotator cuff during operation.

The technique has been validated and provides robust data on the microcirculation. ${ }^{18-23}$ Factors affecting the measurements ${ }^{24}$ include temperature, external pressure, stress, ambient light and movement artefact. It is important to be aware of these variables so that appropriate precautions can be taken during measurements.

In considering 60 readings from ten control cuffs, there was no statistically significant variation in flux within the six areas measured ( $t$-test, $\mathrm{p}=0.2370)$, and therefore the so-called critical zone of hypoperfusion in the rotator cuff was not demonstrated. It is questionable whether a larger study might demonstrate a significant difference. The results of our analysis were not borderline as a p-value of 0.2370 is far above the significance level of 0.05 . Therefore, it is unlikely that there would be significance even in a larger group.

We demonstrated significant differences between the mean flux in normal and diseased cuffs. This was lower in those with impingement than in normal cuffs $(p=0.0196)$ and these preliminary laser Doppler flowmetry recordings agree with the current knowledge of the vascularity of the pathological cuff. . $^{55-27}$

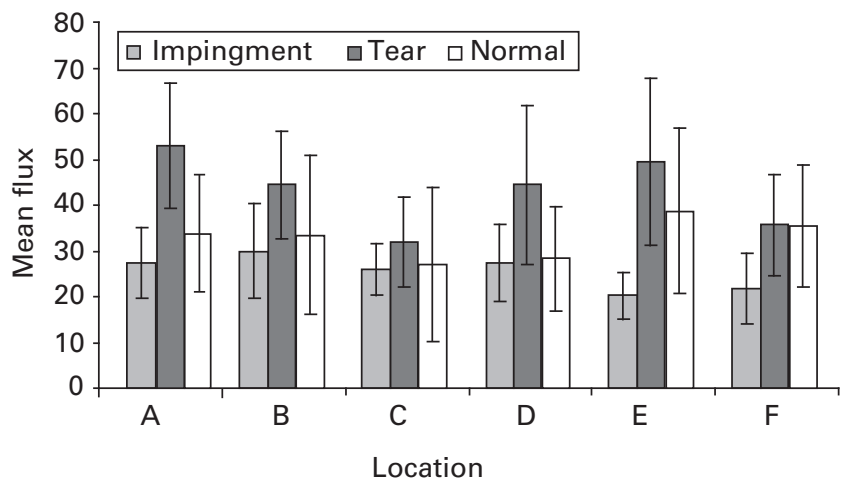

Fig. 4

Mean and 95\% confidence interval for laser Doppler flowmetry flux for impingement, tear and normal cases by anatomical location (see Table II).

The flux was significantly higher at the edges of the tear in torn cuffs than in normals $(\mathrm{p}=0.0089)$. This may represent increased vascularity due to a process of repair. We emphasise that massive cuff tears were excluded from the study as we were unable to measure the flux reliably in such cases without dissecting and mobilising the tissue of the cuff, which would have affected the flux. Matthews et $\mathrm{al}^{28}$ demonstrated that vascularity and healing potential are reduced in massive tears (grade $4^{10}$ ), which were excluded from our study.

We found that the laser Doppler flowmetry flux was significantly lower in cuffs with impingement than in those with tears $(p<0.0001)$. Codman, ${ }^{29}$ on the basis of his cadaver studies and operative findings, described the critical area as the anterior corner of the supraspinatus tendon near its insertion, where it is prone to rupture and is the site of calcium deposits. His work was validated by subsequent cadaver perfusion studies ${ }^{6,24,30-32}$ that demonstrated an area of hypovascularity, and implicated this in the pathogenesis of cuff tears. ${ }^{26,33}$

Brooks et $\mathrm{al}^{27}$ performed a quantitative histological study of the vascularity of the rotator cuff tendon. They measured all vessels larger than $20 \mu \mathrm{m}$ in diameter, irrespective of whether or not they were perfused, and determined 
a reduction in the number and size of the blood vessels between $10 \mathrm{~mm}$ and $20 \mathrm{~mm}$ from the insertion of supraspinatus. However, they also found an identical vascular critical zone in infraspinatus, which is torn much less frequently. ${ }^{34}$ They questioned the relationship of the critical zone to the aetiology of cuff tears, and concluded that findings based on such studies alone can be misleading.

Conversley, Moseley and Goldie ${ }^{35}$ studied the vascular pattern in the cuff tendons, including the critical zone of the supraspinatus, and found a vascular network that received contributions from the anterior humeral circumflex, subscapular and suprascapular arteries. They concluded that the critical zone was not much less vascularised than other parts of the cuff; rather, it was rich in anastomoses between the osseous and tendinous vessels. Rathbun and Macnab ${ }^{26}$ also studied perfusion both with the arm in full adduction and at $90^{\circ}$ of abduction. They noted that the supraspinatus tendon was compressed as it passed over the head of the humerus in adduction, and that its vessels were occluded, whereas they were satisfactorily perfused with the arm abducted. Rothman and Parke ${ }^{32}$ alluded to a similar 'wringing-out' effect. Our findings support those of Rathbun and Macnab ${ }^{26}$ and Moseley and Goldie. ${ }^{35}$ The normal tendon has uniform flux and, as measured by laser Doppler flowmetry, there is no significant reduction in functional perfusion in the critical zone. This suggests that reduced vascularity is more likely to be an effect rather than the primary cause of cuff disease.

Swiontkowski et $\mathrm{l}^{36}$ performed laser Doppler flowmetry studies on 11 patients during open surgery for rotator cuff disease. They concluded that impingement may produce a hyperaemic response in the cuff, which is contrary to our findings. However, the use of an open approach with detachment of the deltoid may have contributed to their findings, as the surgical trauma may have altered local blood flow. They also noted hyperaemia at the edges of the torn tendon and postulated this as a possible attempt at healing.

Other in vivo methods, such as intravital fluorescence microscopy, require the systemic application of potentially toxic ${ }^{37,38}$ fluorescent contrast medium, for example rhodamine-6G and fluorescein isothiocyanate, and a large, expensive intravital microscope. Because of these limitations, in vivo studies of microcirculation in humans have been restricted to the skin, nail fold and conjunctiva.

Orthogonal polarisation spectral imaging has been used for quantitative assessment of the human microcirculation in arthroscopic surgery ${ }^{36}$ but this requires the insertion of a $12 \mathrm{~mm}$ probe into the subacromial space, which is already compromised in cases of impingement. Also, any quantitative assessment of microcirculatory parameters must be performed from recorded video images. We were unable to demonstrate a functional 'critical zone of hypoperfusion' in the normal tendon. This is probably an artefact of the injection technique in cadavers, where most studies have looked at vessels $>20 \mu \mathrm{m}$ in diameter, whereas laser Doppler flow- metry allows measurements in vessels of $10 \mu \mathrm{m}$, which appear to play a significant role in perfusion.

An in vivo study carries ethical considerations that introduce inevitable limitations. We were unable to age-match the different groups. Patients were only invited if they required an arthroscopic operation for the treatment of their shoulder, and could not be asked to participate for the purposes of the study alone. The control group comprised patients undergoing arthroscopic evaluation for instability in the absence of abnormal pathology of the cuff. Although this control group is younger, it would be ethically unacceptable to obtain age-matched controls, which would involve recruiting 'normal' subjects to undergo an arthroscopic operation.

A major strength of this technique is that it allows realtime output. It could be used in further studies on the rotator cuff, and may help to identify strategies for tendonrepair based on individual patterns of perfusion.

No benefits in any form have been received or will be received from a commercial party related directly or indirectly to the subject of this article.

\section{References}

1. Stevenson JH, Trojian T. Evaluation of shoulder pain. J Fam Pract 2002;51:605-11.

2. McMahon PJ, Sallis RE. The painful shoulder: zeroing in on the most common causes. Postgrad Med 1999;106:36-8, 41-3, 47-9.

3. Spindler KP, Dovan TT, McCarty EC. Assessment and management of the painful shoulder. Clin Cornerstone 2001;3:26-37.

4. Iannotti JP. Evaluation of the painful shoulder. J Hand Ther 1994;7:77-83.

5. Biberthaler $\mathbf{P}$, Wiedemann $E$, Nerlich $A$, et al. Microcirculation associated with degenerative rotator cuff lesions: in vivo assessment with orthogonal polarization spectral imaging during arthroscopy of the shoulder. J Bone Joint Surg [Am] 2003;85A:475-80.

6. Determe $\mathbf{D}$, Rongières $\mathbf{M}$, Kany $\mathbf{J}$, et al. Anatomic study of the tendinous rotator cuff of the shoulder. Surg Radiol Anat 1996;18:195-200.

7. Nilsson GE, Tenland T, Obert PA. A new instrument for continuous measurement of tissue blood flow by light beating spectroscopy. IEEE Trans Biomed Eng 1980;27:1219.

8. Nötzli HP, Swiontkowski MF, Thaxter ST, Carpenter GK 3rd, Wyatt R. Laser Doppler flowmetry for bone blood flow measurements: helium-neon laser light attenuation and depth of perfusion assessment. J Orthop Res 1989;7:413-24.

9. No authors listed. World Medical Association Declaration of Helsinki. http:// www.wma.net (date last accessed 30 May 2008).

10. Levy O, Sforza G, Dodenhoff RM, Copeland SA. Arthroscopic evaluation of the impingement lesion: pathoanatomy \& classification. J Bone Joint Surg [Br] 2000;82B(Suppl 3):233.

11. Nicholls RL, Green D, Kuster MS. Patella intraosseous blood flow disturbance during a medial or lateral arthrotomy in total knee arthroplasty: a laser Doppler flowmetry study. Knee Surg Sports Traumatol Arthrosc 2006;14:411-16.

12. Feke GT. Laser Doppler instrumentation for the measurement of retinal blood flow: theory and practice. Bull Soc Belge Ophtalmol 2006;302:171-84.

13. Myers SI, Wang L, Myers DJ. Loss of renal function and microvascular blood flow after suprarenal aortic clamping and reperfusion (SPACR) above the superior mesenteric artery is greatly augmented compared with SPACR above the renal arteries. J Vasc Surg 2007;45:357-66.

14. Nakashima T, Sone M, Fujii H, et al. Blood flow to the promontory in cochlear otosclerosis. Clin Otolaryngol 2006;31:110-15

15. Sone M, Sato E, Hayashi H, Fujimoto Y, Nakashima T. Vascular evaluation in laryngeal diseases: comparison between contact endoscopy and laser Doppler flowmetry. Arch Otolaryngol Head Neck Surg 2006;132:1371-4.

16. Mani R, Cooper C, Kidd BL, Cole JD, Cawley MI. Use of laser Doppler flowmetry and transcutaneous oxygen tension electrodes to assess local autonomic dysfunction in patients with frozen shoulder. J R Soc Med 1989;82:536-8.

17. Larsson R, Oberg PA, Larsson SE. Changes of trapezius muscle blood flow and electromyography in chronic neck pain due to trapezius myalgia. Pain 1999;79:45-50.

18. Larsson SE, Cai H, Oberg PA. Continuous percutaneous measurement by laserDoppler flowmetry of skeletal muscle microcirculation at varying levels of contraction force determined electromyographically. Eur J Appl Physiol Occup Physio 1993;66:477-82. 
19. Elas M, Ahm KH, Parasca A, et al. Electron paramagnetic resonance oxygen images correlate spatially and quantitatively with Oxylite oxygen measurements. Clin Cancer Res 2006;12:4209-17.

20. Bishai JM, Blood AB, Hunter CJ, Longo LD, Power GG. Fetal lamb cerebral blood flow (CBF) and oxygen tensions during hypoxia: a comparison of laser Doppler and microsphere measurements of CBF. J Physiol 2003;546:869-78.

21. Nwaigwe $\mathbf{C l}$, Roche MA, Grinberg $\mathbf{0}$, Dunn JF. Brain tissue and sagittal sinus p02 measurements using the lifetimes of oxygen-quenched luminescence of a ruthenium compound. Adv Exp Med Biol 2003;530:101-11.

22. Dunn JF, Nwaigwe $\mathbf{C I}$, Roche $\mathbf{M}$. Measurement of arterial, venous, and interstitial p02 during acute hypoxia in rat brain using a time-resolved luminescence-based oxygen sensor. Adv Exp Med Biol 1999;471:43-8.

23. Seddon BM, Honess DJ, Vojnovic B, Tozer GM, Workman P. Measurement of tumor oxygenation: in vivo comparison of a luminescence fibre-optic sensor and a polarographic electrode in the p22 tumor. Radiat Res 2001;155:837-46.

24. Oberg PA. Innovations and precautions. In: Shepherd AP, Oberg PA, eds. Laser-Doppler blood flowmetry. Kluwer Academic Publishers, 1990:93-108.

25. Resch H, Breitfuss H. Spontaneous tendon ruptures: etiology, pathogenesis and therapy. Orthopade 1995;24:209-19 (in German).

26. Rathbun JB, Macnab I. The microvascular pattern of the rotator cuff. J Bone Joint Surg [Br] 1970;52-B:540-53.

27. Brooks CH, Revell WJ, Heatley FW. A quantitative histological study of the vascularity of the rotator cuff tendon. J Bone Joint Surg [Br] 1992;74-B:151-3

28. Matthews TJ, Hand GC, Rees JL, Athanasou NA, Carr AJ. Pathology of the torn rotator cuff tendon: reduction in potential for repair as tear size increases. J Bone Joint Surg [Br] 2006;88-B:489-95
29. Codman EA. The shoulder: rupture of the supraspinatus tendon and other lesions in or about the subacromial bursa. Florida: Krieger-Publishing Company, 1984.

30. Ling SC, Chen CF, Wan RX. A study on the vascular supply of the supraspinatus tendon. Surg Radiol Anat 1990;12:161-5.

31. Lohr JF, Uhthoff HK. The microvascular pattern of the supraspinatus tendon. Clin Orthop 1990;254:35-8.

32. Rothman RH, Parke WW. The vascular anatomy of the rotator cuff. Clin Orthop 1965; $41: 176-86$

33. Fukuda H, Hamada K, Yamanaka K. Pathology and pathogenesis of bursal-side rotator cuff tears viewed from en bloc histologic sections. Clin Orthop 1990;254:7580.

34. Neer CS 2nd. Anterior acromioplasty for the chronic impingement syndrome in the shoulder: a preliminary report. J Bone Joint Surg [Am] 1972:54-A:41-50.

35. Moseley HF, Goldie I. The arterial pattern of the rotator cuff of the shoulder. J Bone Joint Surg [Br] 1963;45-B:80-9.

36. Swiontkowski MF, lannotti JP, Boulas HJ, Esterhai JL. Intraoperative assessment of rotator cuff vascularity using laser Doppler flowmetry. In: Post M, Morr, eds. Surgery of the shoulder. St Louis: Mosby, 1990:208-12.

37. Harris AG, Sinitsina I, Messmer K. Intravital fluorescence microscopy and phototoxicity: effects on leukocytes. Eur J Med Res 2002;117:28-3.

38. Saetzler RK, Jallo J, Lehr HA, et al. Intravital fluorescence microscopy: impact of light-induced phototoxicity on adhesion of flurescently labeled leukocytes. J Histochem Cytochem 1997:45:505-13. 